\title{
A!
}

This is an electronic reprint of the original article.

This reprint may differ from the original in pagination and typographic detail.

Bertinetto, Carlo G.; Vuorinen, Tapani

\section{Influence of Pre-Processing and Distance on Spectral Classification: A Simulation Study}

Published in:

Vibrational Spectroscopy

DOI:

10.1016/j.vibspec.2014.08.005

Published: 01/01/2014

Document Version

Peer reviewed version

Please cite the original version:

Bertinetto, C. G., \& Vuorinen, T. (2014). Influence of Pre-Processing and Distance on Spectral Classification: A Simulation Study. Vibrational Spectroscopy, 74, 110-119. https://doi.org/10.1016/j.vibspec.2014.08.005

This material is protected by copyright and other intellectual property rights, and duplication or sale of all or part of any of the repository collections is not permitted, except that material may be duplicated by you for your research use or educational purposes in electronic or print form. You must obtain permission for any other use. Electronic or print copies may not be offered, whether for sale or otherwise to anyone who is not an authorised user. 


\section{Influence of Pre-Processing and Distance on}

\section{Spectral Classification: a Simulation Study}

3 Carlo G. Bertinetto*, Tapani Vuorinen

4 Department of Forest Products Technology, School of Chemical Technology, Aalto

5 University, P.O. Box 16300, 00076 Aalto, Finland.

6

7 Abstract

8 An investigation on the influence of pre-processing on the recognition of chemically

9 similar areas in a spectral image, using simulated data. Fictitious spectra of mixtures of

10 five components at varying concentrations were corrupted by different types of noise to

11 mimic typical signals from Raman imaging. They were then processed by various

12 combinations of pre-processing functions, including baseline correction, smoothing,

13 normalization and Principal Components (PC) compression, and by two clustering

14 algorithms ( $k$-means and agglomerative hierarchical clustering) to recognize the original

15 mixtures. The clusters obtained by the different pre-processing combinations and distance

16 metrics were evaluated by statistical parameters (Rand index and silhouette coefficient)

17 and visual inspection. Perhaps the best performing on the basis of all considered criteria

18 is the combination using an adaptive polynomial detrending, a slight smoothing,

19 normalization by the total signal intensity and compression by 4 PCs (spanning $80 \%$ of

20 the total variance). More detailed analysis was also carried out on subsets of the whole

\footnotetext{
* Corresponding author. Tel.: +358 947001; fax: +358 947024259. E-mail address: carlo.bertinetto@aalto.fi (Carlo G. Bertinetto).
} 
21 data with a particular type of noise and on the influence of each single pre22 processing/clustering variable.

23 Keywords: Pre-processing; Raman microimaging; Cluster Analysis; Simulated spectra;

24 Rand index

\section{Introduction}

Studies involving spectroscopic measurements are increasingly coupled with computational methods to extract more information from the often huge amount of data that can be produced by nowadays instruments. The most advanced analysis and prediction techniques, such as multivariate modeling, curve resolution and machine

31 learning, usually require a very high data quality to reach their full potential, e.g. detecting very small components or grasping complex and subtle relationships. Whenever this requirement is not achievable, the analysis is commonly preceded by pre-processing, a

34 term that comprises several operations to remove non-relevant sources of variation, instrumental artifacts and/or non-linear behavior [1]. These operations can be very diverse and depend on the specific type of spectroscopy and scope of the investigation. For example, IR spectra are often subject to smoothing, derivatization, multiplicative scatter correction and wavenumber selection, whereas mass spectra can undergo alignment, dead time correction or de-isotoping. Comprehensive reviews of pre-processing methods can

40 be found elsewhere [1-4]. Several software packages, such as Unscrambler, Grams, 41 Matlab, R and SIMCA, as well as software built-in with spectroscopic instruments, offer 42 a wide range of pre-processing tools.

43 It is commonly assumed that pre-processing improves the spectral analysis and leads to 44 more accurate, simple and robust models [5]. However, if not performed in the right way, 
45 it can also introduce unwanted variation that will influence all the following steps of the analysis and may thus hamper the successful outcome of the entire experiment [2]. The choice of an optimal pre-processing strategy is thus critical, but little systematic study has so far been carried out to provide clear guidelines on when to use or to avoid certain methods or combination of methods. Users often make these decisions without following objective criteria, a risky approach considering that the effects of each numerical preprocessing step are usually not transparent when dealing with large data sets. Moreover, recent research [2] produced results that contrast the conventional wisdom on the matter, stressing the need for a deeper understanding.

Probably the biggest hurdle in conducting an exhaustive investigation on pre-processing is the huge dimension of the variable space when taking into account all methods, parameters, data characteristics and goals of the analysis. Much of the acquired knowledge is scattered in the literature among many works, since researchers often try a few different pre-processing options any time they conduct a chemometric study. Such knowledge is fragmented, occasionally conflicting and too case-related to be of valuable use for other scientists. Works that address the issue more systematically can be found on IR $[1,2,5-8]$ and some on Raman $[9,10]$ spectroscopy. They all optimize pre-processing on a supervised modeling problem, either a multivariate regression or a PLS-based classification, usually with 2 classes. Engel et al. [2] identify three main approaches for

64 the assessment and selection of a pre-processing strategy: (i) trial and error; (ii) visual inspection; (iii) data quality metrics. The first approach, in which different strategies are applied and the best performing according to the analysis goal is selected, is the most

67 robust and reliable, but can sometimes be computationally too expensive for a practical use. In the second one, the pre-processed data are inspected by simply looking at them 
and checking if any artifacts are still visible. It is a simple approach and thus widely employed, but it is subjective and often inaccurate. The third approach consists of using data quality metrics to quantify the presence of artifacts in the data; it is a useful compromise between the former two, although suitable metrics are not always available and they are nevertheless dependent on the specific analysis. A few authors $[5,7,10]$ combined the trial-and-error approach with genetic algorithms to find the best combination of pre-processing functions among a wide range of possibilities. They found optimal or near-optimal solutions in a relatively short time, but provided little or no interpretation of the effects of the considered operations on the final outcome. In some cases, they even suggest discarding well-performing solutions that are deemed "odd" in favor of nearly equally good ones that can be more easily explainable by prior knowledge $[5]$.

The aim of the present work is to investigate the influence of some important preprocessing functions on a problem of unsupervised spectral recognition using more than two classes. This problem has received less attention than the supervised sort, but it is frequently encountered in many applications ranging from the classification of drugs [11] to the identification of regions of interest in hyperspectral images $[12,13]$. The present analysis is done on simulated spectra, an approach which does not guarantee absolute fidelity to the physical world, but which allows for a more precise evaluation of each variable. In particular, the data are made to resemble Raman microimaging spectra, including their typical noise and distortions, such as broad sloping baselines caused by fluorescence of the sample, Gaussian noise and peak height differences caused by variations in laser intensity or in sample depth $[10,14,15]$. Some of these effects occur in other types of spectroscopy as well; the results of this study can therefore be partly 
93 generalized. Cosmic radiation, a common type of noise affecting all charge-coupled

94 device detectors, is not taken into account here because its correction is relatively easy to

95 do and evaluate, even visually $[16,17]$. The pre-processing functions and parameters

96 considered here are restricted to a limited number of widely used options, with their order

97 defined beforehand according to sensible principles, to keep the variable space within a

98 manageable size. The scope of this paper is indeed to provide broad guidelines rather than

99 seeking the best possible strategy, which is usually dependent on the specific context.

100 Nevertheless, this investigation includes the most important pre-processing operations for

101 this kind of data.

102 The simulation experiment consists of: (i) defining fictitious spectra of mixtures of five

103 components at varying concentrations and corrupted by different types of noise; (ii)

104 processing them by combinations of selected functions for noise removal and data

105 compression; (iii) recognizing the original mixtures by clustering algorithms [18], namely

$106 k$-means clustering and agglomerative hierarchical clustering. Clustering algorithms,

107 which perform an unsupervised classification of data, have already been employed in

108 several works on spectral imaging, both as a main analysis tool [19-21] and as a treatment

109 prior to other techniques [22,23], but to our knowledge this is the first systematic study

110 about the influence of pre-processing on the clustering of spectra. The results are

111 discussed in detail by statistically evaluating the clustering accuracy, on the whole data

112 set as well as subsets with a particular type of noise, and by examining trends among each

113 pre-processing/clustering variable.

114

115 2. Data set and method

116 2.1. Simulated spectra 
117 Five 'pure component' spectra, indicated by the letters A to E, were defined as vectors of

1181000 elements containing sums of various Lorentzian curves convoluted by a Gaussian

119 (amplitude $=1$; standard deviation $=5$ points; ) and normalized by their Euclidean norm.

120 These five spectra, plotted in Fig. 1a, were linearly combined into 'mixture' spectra with

12110 different proportions and normalized by their amplitude ([max $-\min ])$. Figure $1 b$

122 shows a histogram of these proportions, from which it can be observed that component A

123 imitates a 'background' substance present in large amounts everywhere in the sample (as

124 could be an embedding medium), while E represents a 'trace' compound. These mixtures

125 can be visually grouped into 4 classes: three with a higher abundance of A, B and C,

126 respectively, and a fourth with roughly equal concentrations of components A-D. This

127 partition is confirmed by $k$-means clustering both on the mixture spectra and on the

128 concentration matrix, using either Euclidean, city-block or cosine distance.

129 Each of these ten spectra was added a baseline distortion and shot noise. The baseline

130 distortion could be a straight line decreasing from 1 to 0 over the abscissa range, or a $3^{\text {rd }}$

131 order polynomial intercepting values of $0,0.5,1$ and 0.7 at vector elements $n .1,200,700$

132 and 1000, respectively. Each baseline was multiplied by $0,0.5,1,2,4$ or 8 to modify its

133 baseline-to-signal ratio (BSR). Shot noise was taken from five measurements in air by an

134 Alpha300 R Confocal Raman microscope (Witec GmbH, Germany), out of which a

135 section of 1151 spectral points was selected, encompassing the range between 637 and

$1363240 \mathrm{~cm}^{-1}$. They were mean-centered and sigma-scaled, with sigma derived by a Gaussian

137 fit of their probability density function, approximated by their histogram plot. 31 vectors

138 of 1000 elements were then carved out of every 1151-long vector by choosing different

139 starting points. The number of these vectors was then increased four-fold by reflection on

140 the $\mathrm{X}$ or $\mathrm{Y}$ axis or both, giving a total pool of 620 noise vectors. These were randomly 
141 assigned to the mixture spectra and divided by $100,70,30,20,10$ or 5 to modify their

142 signal-to-noise ratio (SNR). Among the most noisy of the resulting spectra, two sharp

143 peaks deriving from oxygen and nitrogen Raman signals were visible. These peaks were

144 considered useful for the simulation because they look like leftovers from an imperfect

145 cosmic ray removal, a rather frequent case in Raman image analysis. The previous

146 translations and reflections of the noise vector ensure that these peaks do not occur always

147 at the same positions and thus have a more random-like behavior.

148 Finally, each baseline- and noise-added spectrum was appropriately multiplied to set their

149 Euclidean norm to 1, 2, 4 or 8 . The total number of simulated spectra is 2640 and all the

150 parameters that define them are listed in Table 1. A few examples of the final noise-added

151 spectra are depicted in Fig. 2.

152

153

\subsection{Pre-processing}

154 The pre-processing consisted of four steps: baseline treatment, smoothing, normalization 155 and Principal Component (PC) compression [24], performed in this order except where 156 specified below. The baseline was treated either with an adaptive detrending or with a 157 derivatization. The former is an in-house written method [25] consisting of $5^{\text {th }}$ order 158 polynomial iterative fitting of the peak-free spectral points recognized by an algorithm 159 based on the continuous wavelet transform. The latter is a Savitzky-Golay $2^{\text {nd }}$ derivative 160 using a 21-point window and a $2^{\text {nd }}$ order polynomial. The smoothing method was either 161 a Whittaker smoother [26], using a $2^{\text {nd }}$ derivative and a smoothness parameter $\lambda=100$, or 162 a wavelet-based smoothing obtained by setting to zero all the detail coefficients from a 163 decomposition at level 3 using the discrete Meyer wavelet. This wavelet was chosen 164 according to the energy/entropy criterion among a set of 45 wavelets including the 
165 Daubechies, Symlet and Coiflet families [27]. Such smoothing method is expected to be 166 more specifically adapted to these instrumental noise patterns. The normalization could

167 be either according to the 1-norm or to the 2-norm, also known as city-block and 168 Euclidean norm, respectively. The number of retained PCs was chosen to span either 90\% 169 or $80 \%$ of the total variance in the data matrix; this number can be very different 170 depending on the other pre-processing choices.

171 When the baseline was treated with a $2^{\text {nd }}$ order derivative, smoothing was carried out first, 172 to avoid noise amplification caused by derivatization. The numerical parameters used for 173 baseline correction and smoothing were determined by visual inspection on a few sample

174 spectra and by comparison with other works in the literature that are more focused on this 175 aspect $[5,9,10]$. As mentioned in the introduction, the purpose of this study is to evaluate main pre-processing approaches rather than do a very detailed optimization.

\subsection{Cluster analysis}

179 Clustering is performed by two commonly used methods: $k$-Means Clustering (KMC) and 180 Agglomerative Hierarchical Clustering (AHC) [18]. In KMC, $k$ clusters are each represented by a centroid in the spectral space and every data point (in the present case: every spectrum) is assigned to the nearest centroid. The centroids are then recalculated by averaging the elements of each cluster and all data points are reassigned to these new

184 centroids; this step is repeated until the centroid positions do not change. Because the 185 result is influenced by the random initialization of centroids, the algorithm was repeated 1861000 times with different initial conditions and the solution with the lowest sum of squared errors (SSE) was retained. Because such SSE value was reached in more than half of the repetitions in almost all simulations and because trials with a particle swarm 
optimizer could not improve this outcome, it was considered as a reasonable approximation of the optimal SSE. The number of clusters $k$ was set as either 4 or 10 ;

191 distances were calculated according to the city-block metric, the Euclidean metric or

192 based on the cosine angle between data points.

193 By contrast, AHC starts by considering every data point as a separate cluster, then merges

194 the two clusters with minimum mutual distance and repeats this operation until a single cluster remains. In addition to a distance metric, HCA needs a criterion to determine the distance between clusters: Ward's method was used here, for which the distance is

197 defined as the increase in SSE when two clusters are merged. It is somewhat similar to

198 SSE-optimized KMC, with the difference that the SSE is minimized for single merging 199 steps instead of the final clusters. This method is widely employed because of its accuracy and robustness to noise; indeed, preliminary trials on our data showed that it performed clearly better than other widely used criteria, such as single, complete and average linkage. Because Ward's method is typically formulated in terms of Euclidean distance, only this metric was used for AHC simulations. In AHC the number of clusters does not need to be determined in advance, but in order to have a more uniform comparison with $\mathrm{KMC}$, the merging was stopped when 4 and 10 clusters were formed, respectively (the final number of clusters will be denoted as $k$ for AHC simulations as well).

207 All combinations of the mentioned pre-processing and clustering variables were tried, for 208 a total of 648 simulations. All the employed simulation options (including the one of 209 doing nothing) and the abbreviations used to indicate them in the following sections are summarized in Table 2. The calculations were performed using MATLAB® version 8.2

211 R2013b (The Mathworks, USA). 


\section{Results and discussion}

\subsection{Overall evaluation}

215 The pre-processing combinations are primarily evaluated by the accuracy of the resultant 216 clusters. As secondary evaluation criteria, the cluster separation, some computational

217 issues and the performance on certain subsets of the original data are also considered here.

218 The results with 4 and 10 clusters are treated separately because they correspond to two 219 different and not fully comparable problems: the former mimics the typical operation of identifying chemically similar (though not identical) points in an image, whereas the latter is a more demanding task meant to test the capability of the pre-processing methods in discerning the exact mixture.

223 The clustering accuracy is quantified by the Rand index $(R)$ [28], which measures how a clustering matches a known set of classes, in the present case either the original mixtures or the four classes indicated in Fig. 1b. $R$ (formula shown in Eq. A.1, Appendix A) can have values between 0 and 1 , with 1 corresponding to a perfect cluster-class match. The fifteen pre-processing/clustering combinations that yielded the highest $R$ for both KMC and $\mathrm{AHC}$ are ranked and listed in Table 3; in the following paragraphs they will often be referred to with their $R$-wise rank number. For KMC, the best ones are $\{k=4$, WPD, NoS, $2 \mathrm{~N}, 90 \%[76], \mathrm{CB}\}$ and $\{k=10, \mathrm{WPD}, \mathrm{NoS}, 1 \mathrm{~N}$, noPCA, cos $\}$, respectively (see Table 2

231 for abbreviations). AHC generally yielded a lower $R$ than KMC, although for a few 232 combinations it was similar or higher. The best ones are $\{k=4$, WPD, WavS, $1 \mathrm{~N}, 80 \%$ $233[4]\}$ and $\{k=10$, WPD, WavS, $2 \mathrm{~N}, 80 \%[4]\}$, which correspond to the $7^{\text {th }}$ and $3^{\text {rd }}$ best outcome for KMC with 4 and 10 clusters, respectively. For both KMC and AHC, the next

235 few combinations in the ranking after the first have an $R$ value almost as good, suggesting 236 that they also are reasonable choices. 
237 The pre-processing function that appears almost everywhere in Table 3 is WDP; other 238 functions are frequent for certain calculations: CB (appearing in the first ten combinations 239 for $\mathrm{KMC}, k=4$ ), $80 \%$ (in the $2^{\text {nd }}$ to $7^{\text {th }}$ combination for $\mathrm{KMC}, k=10$ ), $1 \mathrm{~N}$ (in the first 240 four combinations for $\mathrm{AHC}, \mathrm{k}=4$ ) and $2 \mathrm{~N}$ (in the first five combinations for $\mathrm{AHC}, \mathrm{k}=$ 241 10). For KMC, some of the best clusterings (including the very best) are obtained without 242 any smoothing, whereas all the best clustering by AHC have either WavS or WS. This is 243 probably due to the stepwise and non-revocable definition of clusters by the AHC 244 algorithm, which implies that any erroneous initial assignment of a noisy point cannot be 245 corrected later. No particular prevalence or trend is observed for the remaining pre246 processing functions.

247 Another meaningful evaluation criterion is cluster separation, especially useful to refine 248 the analysis among equally accurate clusterings. A higher separation increases the 249 probability that the result is robust and not out of chance from these particular data. The 250 separation is quantified by the average silhouette coefficient $(S)$ [29] over all data 251 (formula shown in Eq. A.2, Appendix A), whose value ranges between -1 and 1 for 252 perfectly undistinguishable and perfectly separated clusters, respectively. $S$ for the most 253 accurate clusterings is indicated in Table 3 next to the $R$ value. For KMC, some of the 254 clusterings with the best $R$, including the very best, do not score well according to the $S$ 255 criterion, as can be visualized by the $S$ vs $R$ plot in Fig. 3. Following the $R$-based ranking, 256 the first results with $S>0.5$ are the $7^{\text {th }}$ for $k=4$ (highlighted by a circle in Fig. 3) and 257 those from $2^{\text {nd }}$ to $7^{\text {th }}$ for $k=10$. On the other hand, the clusters from AHC, especially the 258 most accurate ones, have a much higher $\mathrm{S}$, probably as a result of the characteristics of 259 the AHC algorithm mentioned above. 
260 It is worth noticing that some of the best combinations in Table 3 don't use any PC 261 compression. Although this choice may not be detrimental towards the quality of the 262 results, in some contexts it can be unpractical because of the required computation time, 263 approximately 100 times longer than when using PCA. It may therefore be convenient to 264 use equally good ( $R$-wise) pre-processing combinations that include PCA when dealing 265 with large data sets. Another interesting observation is that some of the best results 266 obtained in these simulations come from a number of PCs lower than 5, which is the 267 actual number of independent components known to be in the data. This fact suggests that 268 such pre-processing strategies were not able to recover all the information out of the 269 added noise or that they might have oversimplified the data, as is qualitatively illustrated 270 in the example in Fig. 4, which plots the first 6 PCs after WPD, WavS and 1N. All the 271 features of pure component spectra can be observed in these PCs, including those of the 272 'trace' component E, indicated by ovals. However, several noise-related features, 273 highlighted by shadings, are hardly distinguishable from those of component E: any 274 cutoff in the number of PCs will therefore either exclude E or include unwanted noise.

275 Further information can be obtained by visual inspection. Figure 5 depicts some selected 276 clusterings as color maps: the data points from the same mixture are aligned in rows, with 277 the SNR decreasing in blocks going from left to right; a perfect clustering appears as 278 homogeneous horizontal stripes. For $k=4$, the most accurate results from both KMC and 279 AHC match all the correct classes except for a few noisy data points, see Fig. 5a (only 280 KMC shown). On the other hand, the clustering with highest $R$ for $k=10$ does not 281 correctly distinguish all mixtures and, as can be seen in Figs. 5b (KMC) and 5c (AHC), 282 at least two pairs of mixtures are always clustered together even on data with little noise. 283 Exceptions to this rule are the $6^{\text {th }}$ best clusterings by both $\mathrm{KMC}$ and $\mathrm{AHC}$ and the $7^{\text {th }}$ best 
by KMC (shown in Fig. 5d), which cluster together only mixtures 2 and 3, at least on data with high SNR. The below-optimal $R$ value of these last-mentioned clusterings is caused by a poor performance on noisy data, especially for mixtures 1-3.

These tendencies, i.e. better clustering of noisy data by the pre-processing combinations ranked $1^{\text {st }}$ to $5^{\text {th }}$ in Table 3 (10 clusters) and of clean data by $\{\mathrm{WPD}, \mathrm{WhS}, 1 \mathrm{~N}, 80 \%[3]$, Eucl/CB , are observed even when $k$ is increased up to 15 (not shown here). The choice of optimal pre-processing is thus dependent on the type of data, with the $6^{\text {th }}$ combination (or $7^{\text {th }}$ for $\mathrm{KMC}$ ) seeming more appropriate when the SNR is high. In practice, it is even more so considering that it is usually preferred to have very reliable results on clean data and that the noisiest signals are often discarded as outliers. Moreover, the combination

$294\{$ WPD, WavS/WhS, 1N, 80\%[4], Eucl/CB $\}$ is probably the most interesting because it obtains good clusterings in all four simulations: $7^{\text {th }}$ best for $\{\mathrm{KMC}, k=4\}$ (and, as already observed, best among those with $\mathrm{S}>0.5), 1^{\text {st }}$ for $\{\mathrm{AHC}, k=4\}$ and $6^{\text {th }}$ for $\{\mathrm{KMC} / \mathrm{AHC}$, $k=10\}$ (with the best clustering of low-noise data). Hence, this pre-processing seems a more general data treatment suitable for more than one analysis task.

299 A peculiar result is observed for $\{\mathrm{KMC}, k=4$, WPD, WhS, N2, 80\%[4], CB $\}$, which has 300 the $21^{\text {st }}$ highest $R$ with 4 clusters, shown in Fig. 5e. For the high-SNR data points, mixture n. 9 is (wrongly) clustered together with mixtures n. 6-7, but it is instead clustered correctly for the noisy points. No satisfactory explanation was found and this outcome 303 might be product of chance.

\subsection{Results on subsets of data}

306 To provide a more detailed picture of the clusterings obtained in our simulations, the Rand 307 index was calculated for subsets of the data, which included: (i) spectra with low SNR (= 
$30830,20,10,5)$; (ii) spectra with high $\operatorname{BSR}(=2,4,8)$; (iii) spectra with linear baselines

309 only; (iv) spectra with polynomial baselines only. Figure 6 plots the Rand statistics of the

310 same pre-processing/clustering combinations shown in Table 3, for all data as well as the

311 mentioned subsets. The series with low SNR is always considerably below the overall $R$,

312 especially for AHC, see Figs. 6c and 6d. Particularly notable are the low values for the

$3136^{\text {th }}$ and $7^{\text {th }}$ combination with $k=10$ for both $\mathrm{KMC}$ and $\mathrm{AHC}$, which indicate a poor

314 clustering of noisy data as was observed visually in Fig. 5d. The high-BSR series stays

315 slightly below the overall $R$ most of the times for KMC (Figs. 6a and 6b), whereas for

316 AHC it is more erratic. Subsets with linear or polynomial baseline often have different $R$,

317 but there is no regularity over which outperforms the other. Considering that the baseline

318 correction method (WPD) is the same for all but one of the plotted combinations, the

319 spectral recognition with respect to the baseline type is clearly influenced by the other

320 pre-processing functions as well.

321

322 3.3. Dependence on single pre-processing functions

323 To gain more insight on the effect of each pre-processing/clustering parameter, trends in 324 the $R$ and $S$ statistics for the whole data set were examined on sections of the solution space in which only a single variable was changed at a time. For KMC, 81 sections were

326 produced for each operation (baseline correction, smoothing, normalization, PC 327 compression and clustering with different distance metrics) and number of clusters (4 and 328 10), for a total of 810. For AHC, 27 sections were produced for each operation (which 329 did not include the choice of distance metric), for a total of 216 . Due to the high number 330 and redundancy of these trends, the ones with low $R$ values are ignored from the analysis 331 and only the most representative are shown in Fig. 7. Moreover, since practically the same 
trends were observed for $\mathrm{KMC}$ and $\mathrm{AHC}$, all examples are taken from the former except where specified. It must be pointed out that it is not possible to perfectly separate the effect of one pre-processing function from the other, as was already noticed when commenting about baseline types at the end of Section 3.2. Another evident example is the calculation of the Savitzky-Golay $2^{\text {nd }}$ derivative, which has an influence on shot noise as well.

\subsubsection{Dependence on the baseline correction}

340 The $R$ trend is WPD $>$ S2D $>$ noBC in nearly all cases, see Fig. 7a (crosses), as hinted

341 also by the quasi-absence of S2D in Table 3. An exception are the trends using $k=4$,

$342 \mathrm{WhS}$, noN/1N/2N, noPCA and cos, which are $\mathrm{S} 2 \mathrm{D}>\mathrm{WPD}>$ noBC, see Fig. 7a 343 (triangles). As is already widely known, these numbers show that baseline correction is very important for a good spectral recognition. More debated is whether detrending is better than derivatives and, according to these results, it is. This outcome, also reinforced by the fact that in a few spectra the detrending was not optimal, suggests that derivatives remove more information than was previously thought. Moreover, spectra treated with S2D need many more PCs (about 4-5 times more) to span the same variance, implying that derivatization leads to an unnecessary increase in the problem's complexity.

\subsubsection{Dependence on the smoothing}

352 Smoothing has a very small influence on the clustering accuracy and all tried options

353 produce roughly the same $R$, see Fig. $7 \mathrm{~b}$ (triangles). The only exception is with combinations that yield a high $R$ by AHC, for which WavS $\approx \mathrm{WhS}>\mathrm{NoS}$ (see Fig. 7b, 
considerable increase in $S$, see Fig. 7 b (filled blue circles), and great reduction (up to eight-fold) of the number of PCs needed to describe the same variance in not-smoothed data. Contrary to the expectations about using a wavelet adapted to the noise patterns, no significant difference is observed between applying WhS and WavS. From these results, smoothing does not appear to be absolutely necessary for the analysis task, but it can make the data less blurred and the clustering more simple and stable.

It is important to point out that in the present simulation this operation was performed with the help of background knowledge about peak shapes. In practice, when such knowledge is not available it is better to smooth only to a minimum degree. The small gains derived from an optimal smoothing are usually not worth the risk of oversmoothing and consequent loss of information.

\subsubsection{Dependence on the normalization}

The $R$ trends show that normalization is extremely important for an accurate clustering, see Fig. 7c (crosses). The only exception are the simulations that use noN, noPCA and cos, see Fig 6c (circles), because the cosine distance levels off the differences in total intensities, However, the use of PCA and cosine distance without normalization severely distorts the spectral information, see Fig. 7c (squares). It must be stressed that in this analysis spectra are recognized on the basis of the relative signal proportions; if the absolute signal intensities were more meaningful, other types of normalization (such as internal standards) or none at all would be preferable. For $k=4$, most of the $R$ trends have $1 \mathrm{~N}>2 \mathrm{~N}$, see Fig. 7c (triangles); for $k=10$ no significant difference is observed between the two normalization methods. 
379 In the literature, the Euclidean normalization $(2 \mathrm{~N})$ is employed more often, but there are

380 no clear opinions about which one is better. Out of common sense, if the purpose is to

381 normalize by the total spectral intensity the 1-norm is more appropriate because it is more

382 directly related to the number of interacting photons, but the 2-norm should be preferable

383 for noisy spectra because it gives less weight to small noise peaks. To seek some evidence

384 of this reasoning among our results, we computed the average $R$ using $1 \mathrm{~N}$ and $2 \mathrm{~N}$,

385 respectively, for all data as well as for the low-SNR subset introduced in Section 3.2. The

386129 combinations with lowest $R$, for both the 4-cluster and 10-cluster rankings, were

387 excluded from this average. With $k=4$, these values were $0.8638(1 \mathrm{~N})$ and $0.8623(2 \mathrm{~N})$

388 for all data and $0.8366(1 \mathrm{~N})$ and $0.8434(2 \mathrm{~N})$ for low-SNR data. With $k=10$, they were

$3890.8966,0.9005$ and $0.8857,0.8929$, respectively. In both cases the difference $R(2 \mathrm{~N})-$

$390 R(1 \mathrm{~N})$ is greater for noisy data than for the whole data set; this is in line with our

391 hypothesis, although these differences are too small to be an unequivocal proof.

392

393 3.3.4. Dependence on the compression with principal component analysis

394 PC compression does not have any significant influence on the $R$ values, see Fig. 7d 395 (crosses), except for the already mentioned sharp decrease when using PCA, cosine 396 distance and no normalization. A similar effect is observed also for simulations using $k=$ $3974, \mathrm{~S} 2 \mathrm{D}, 1 \mathrm{~N} / 2 \mathrm{~N}$, cos, see Fig. 7d (triangles). There is no great difference in $R$ between 398 spanning $80 \%$ and $90 \%$ of the total variance. On the other hand, the $S$ plots always follow 399 the trend $80 \%>90 \%>$ noPCA, see Fig. $7 \mathrm{~d}$ (filled blue circles), indicating that a limited 400 number of PCs leads to a better cluster separation. The determination of the exact number 401 of PCs for best separation is out of the scope of this paper.

402 
404 The distance metric presents the most various $R$ trends: $\mathrm{CB} \approx$ Eucl $>\cos$ for $k=4$, 405 WPD, 1N (Fig. 7e, crosses); cos >> Eucl > CB for $k=4$, WhS, S2D, 1N/2N, noPCA (Fig. $4067 \mathrm{e}$, triangles); $\mathrm{CB}>\mathrm{Eucl} \approx \cos$ for $k=4$, WDP, noS, $1 \mathrm{~N} / 2 \mathrm{~N}$ (Fig. 7e, circles); no

407 significant dependence is observed for the other series. For $k=10$, the trends are similar 408 but much less pronounced, often nearly constant. The $S$ trend is always cos $>$ Eucl $>$ CB 409 (Fig 6e, filled blue circles) for both 4 and 10 clusters, which comes in favor of using 410 cosine distance if it is not in conflict with other pre-processing choices.

\section{Conclusion}

413 The presented investigation explored the influence of commonly used pre-processing operations on the problem of chemical recognition from spectral data based on the proportion of its constituting signals. The analysis was carried out through $k$-means and agglomerative hierarchical clustering on a set of spectra that simulate a Raman image.

417 The results were evaluated by visual inspection and statistical parameters, with the aim 418 of providing guidelines for future studies and applications.

419 As expected, no simple relationship was found and the optimal pre-processing combination depends on the type of data and evaluation criteria. A few combinations have

421 been identified as the best choices that produce the most accurate clustering according to 422 the a-priori known chemical mixtures. In particular, the one using an adaptive polynomial 423 detrending [25], some smoothing, normalization by the total intensity and compression by 4 PCs (representing $80 \%$ of the total variance) simultaneously achieved excellent cluster accuracy and separation, computational speed and range of applicability. 
426 Nevertheless, it is not the best performing for very noisy spectra, as shown by color plots 427 and statistics on subsets of the whole data.

428 The analysis of individual pre-processing functions revealed some interesting trends. 429 Baseline correction is very important for an accurate clustering and the employed 430 detrending nearly always outperforms $2^{\text {nd }}$ derivatization in terms of both cluster accuracy 431 and problem simplicity (i.e. it required less PCs). On the other hand, smoothing has little 432 influence on the accuracy, but it improves separation and simplicity. Normalization is necessary for this type of spectral recognition, except for the case using cosine distance

434 and no PC compression. Using the 2-norm appears slightly better than 1-norm for noisy 435 data.

436 PC compression does not have any significant effect on the cluster accuracy, but the separation is best when selecting a not too high number of PCs, here represented by the one spanning $80 \%$ of the data variance. A more complicated dependency is observed for the clustering distance metric, investigated only for the $k$-means method. The Euclidean

440 and city-block distances are equally accurate in many cases, though the former seems 441 slightly favored for coarser clustering (i.e. low number of clusters). The cosine distance 442 yields the highest cluster separation but is accurate only with certain pre-processing 443 choices.

444 The results shown in this paper, although they do not exhaustively cover the whole issue, 445 offer a clearer picture of which pre-processing functions and distances improve the 446 analysis of spectral images and which should instead be avoided.

\section{Acknowledgements}

449 The financial contribution by the Academy of Finland is gratefully acknowledged. 
451 Appendix A: formulae of statistical parameters used for clustering evaluation

452 Rand index $(R)$ :

$$
R=\frac{f_{00}+f_{11}}{f_{00}+f_{01}+f_{10}+f_{11}}
$$

453 where:

$454 f_{00}=$ number of pairs of data points having a different class and a different cluster

$455 f_{01}=$ number of pairs of data points having a different class and the same cluster

$456 f_{10}=$ number of pairs of data points having the same class and a different cluster

$457 f_{11}=$ number of pairs of data points having the same class and the same cluster

458

459

Silhouette coefficient, average value for all data points $(S)$ :

$$
S=\sum_{i=1}^{N}\left(\frac{\mathrm{b}_{i}-\mathrm{a}_{i}}{\max \left(\mathrm{a}_{i}, \mathrm{~b}_{i}\right)}\right) \cdot \frac{1}{N}
$$

460 where:

$461 \quad N=$ total number of data points

462

$\mathrm{a}_{i}=$ average distance from data point $i$ to all other points in cluster $I: i \in I$

463

$\mathrm{b}_{i}=$ average distance from data point $i$ to the points of the neighboring cluster $J: i \notin J$

464

and $\mathrm{b}_{i}$ is minimum among all clusters

465

466 References

467 [1] Å Rinnan, F. van den Berg, S.B. Engelsen, TRAC-Trend. Anal. Chem. 28 (2009)

468 1201-1222. DOI:10.1016/j.trac.2009.07.007.

469 [2] J. Engel, J. Gerretzen, E. Szymańska, J.J. Jansen, G. Downey, L. Blanchet, L.

470 Buydens, TRAC-Trend. Anal. Chem. 50 (2013) 96-106.

471 DOI:10.1016/j.trac.2013.04.015. 
472 [3] S. Castillo, P. Gopalacharyulu, L. Yetukuri, M. Orešič, Chemometr. Intell. Lab. 108 473 (2011) 23-32. DOI:10.1016/j.chemolab.2011.03.010.

474 [4] A. Smolinska, L. Blanchet, L. Buydens, S.S. Wijmenga, Anal. Chim. Acta 750 475 (2012) 82-97. DOI:10.1016/j.aca.2012.05.049.

476 [5] O. Devos, L. Duponchel, Chemometr. Intell. Lab. 107 (2011) 50-58.

477 DOI:10.1016/j.chemolab.2011.01.008.

478 [6] E. Polshin, B. Aernouts, W. Saeys, F. Delvaux, F.R. Delvaux, D. Saison, M. Hertog, 479 B.M. Nicolaï, J. Lammertyn, J. Food Eng. 106 (2011) 188-198.

480 DOI:10.1016/j.jfoodeng.2011.05.003.

481 [7] J. Laxalde, C. Ruckebusch, O. Devos, N. Caillol, F. Wahl, L. Duponchel, Anal. 482 Chim. Acta 705 (2011) 227-234. DOI:10.1016/j.aca.2011.05.048.

483 [8] M. Brandstetter, T. Sumalowitsch, A. Genner, A.E. Posch, C. Herwig, A. Drolz, V. 484 Fuhrmann, T. Perkmann, B. Lendl, Analyst 14 (2013) DOI:10.1039/C3AN00300K.

485 [9] A.T. Weakley, P. Warwick, T.E. Bitterwolf, D.E. Aston, Appl. Spectrosc. 66 (2012) 486 1269-1278. DOI:10.1366/12-06588.

487 [10] T. Bocklitz, A. Walter, K. Hartmann, P. Rösch, J. Popp, Anal. Chim. Acta 704 488 (2011) 47-56. DOI:10.1016/j.aca.2011.06.043.

489 [11] V. Schoonjans, F. Questier, A. Borosy, B. Walczak, D. Massart, B. Hudson, J. 490 Pharm. Biomed. Anal. 21 (2000) 1197-1214. DOI:10.1016/S0731-7085(99)00236-8.

491 [12] M. Miljković, T. Chernenko, M.J. Romeo, B. Bird, C. Matthäus, M. Diem, Analyst 492135 (2010) 2002-2013. DOI:10.1039/C0AN00042F.

493 [13] Q. Zhong, C. Yang, F. Großerüschkamp, A. Kallenbach-Thieltges, P. Serocka, K. 494 Gerwert, A. Mosig, BMC Bioinformatics 14:333 (2013). DOI:10.1186/1471-2105-14495333.

496 [14] P. Heraud, B.R. Wood, J. Beardall, D. McNaughton, J. Chemometr. 20 (2006) 193 497 197. DOI:10.1002/cem.990.

498 [15] O. Stanimirovic, H.F. Boelens, A.J. Mank, H.C. Hoefsloot, A.K. Smilde, Appl. 499 Spectrosc. 59 (2005) 267-274.

500 [16] U.B. Cappel, I.M. Bell, L.K. Pickard, Appl. Spectrosc. 64 (2010) 195-200.

501 [17] H.G. Schulze, R.F. Turner, Appl. Spectrosc. 67 (2013) 457-462. DOI:10.1366/1250206839.

503 [18] A.K. Jain, R.C. Dubes, Algorithms for clustering data, Prentice-Hall, Inc., Upper 504 Saddle River, NJ, USA, 1988. 
505 [19] A.R. Konicek, J. Lefman, C. Szakal, Analyst 137 (2012) 3479-3487.

506 DOI:10.1039/C2AN16122B.

507 [20] I.I. Patel, W.J. Harrison, J.G. Kerns, J. Filik, K. Wehbe, P.L. Carmichael, A.D.

508 Scott, M.P. Philpott, M.D. Frogley, G. Cinque, Anal. Bioanal. Chem. 404 (2012) 1745509 1758. DOI:10.1007/s00216-012-6314-y.

510 [21] J. Piva, J. Silva, L. Raniero, A. Martin, H. Bohr, K.J. Jalkanen, Theor. Chem. Acc. 511130 (2011) 1261-1273. DOI:10.1007/s00214-011-1063-0.

512 [22] P.N. Perera, M. Schmidt, P.J. Schuck, P.D. Adams, Anal. Chim. Acta 702 (2011)

513 172-177. DOI:10.1016/j.aca.2011.06.021.

514 [23] A. Kunstar, J. Leijten, S. van Leuveren, J. Hilderink, C. Otto, C.A. van

515 Blitterswijk, M. Karperien, A.A. van Apeldoorn, J. Biomed. Opt. 17 (2012) (Nov 01, 516 2012) 116012. DOI:10.1117/1.JBO.17.11.116012.

517 [24] H. Abdi, L.J. Williams, Wiley Interdisciplinary Reviews: Computational Statistics 5182 (2010) 433-459. DOI:10.1002/wics.101.

519 [25] C.G. Bertinetto, T. Vuorinen, Appl. Spectrosc. 68 (2014) 155-164.

520 DOI:10.1366/13-07018.

521 [26] P.H.C. Eilers, Anal. Chem. 75 (2003) 3631-3636. DOI:10.1021/ac034173t.

522 [27] R.X. Gao, R. Yan, Wavelets: Theory and applications for manufacturing, Springer, 523 New York, USA, 2011.

524 [28] W.M. Rand, Journal of the American Statistical association 66 (1971) 846-850.

525 DOI:10.1080/01621459.1971.10482356.

526 [29] P.J. Rousseeuw, J. Comput. Appl. Math. 20 (1987) 53-65. DOI:10.1016/0377527 0427(87)90125-7. 

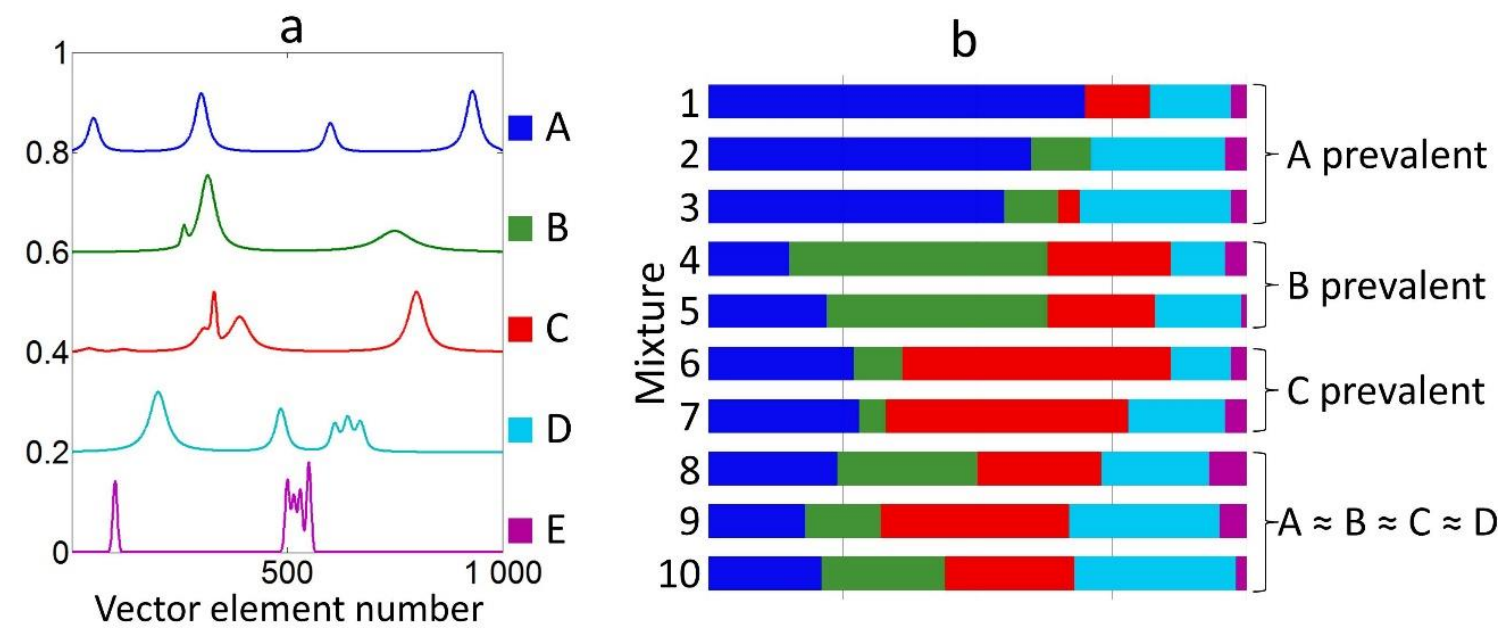

532 Figure 1. Simulated spectra: (a) Spectra of pure components (arbitrary units). (b) Mixture

533 proportions; the parentheses on the right side indicate the classes used for the clustering

534 with $k=4$. For more details on these plots see Table 1. 


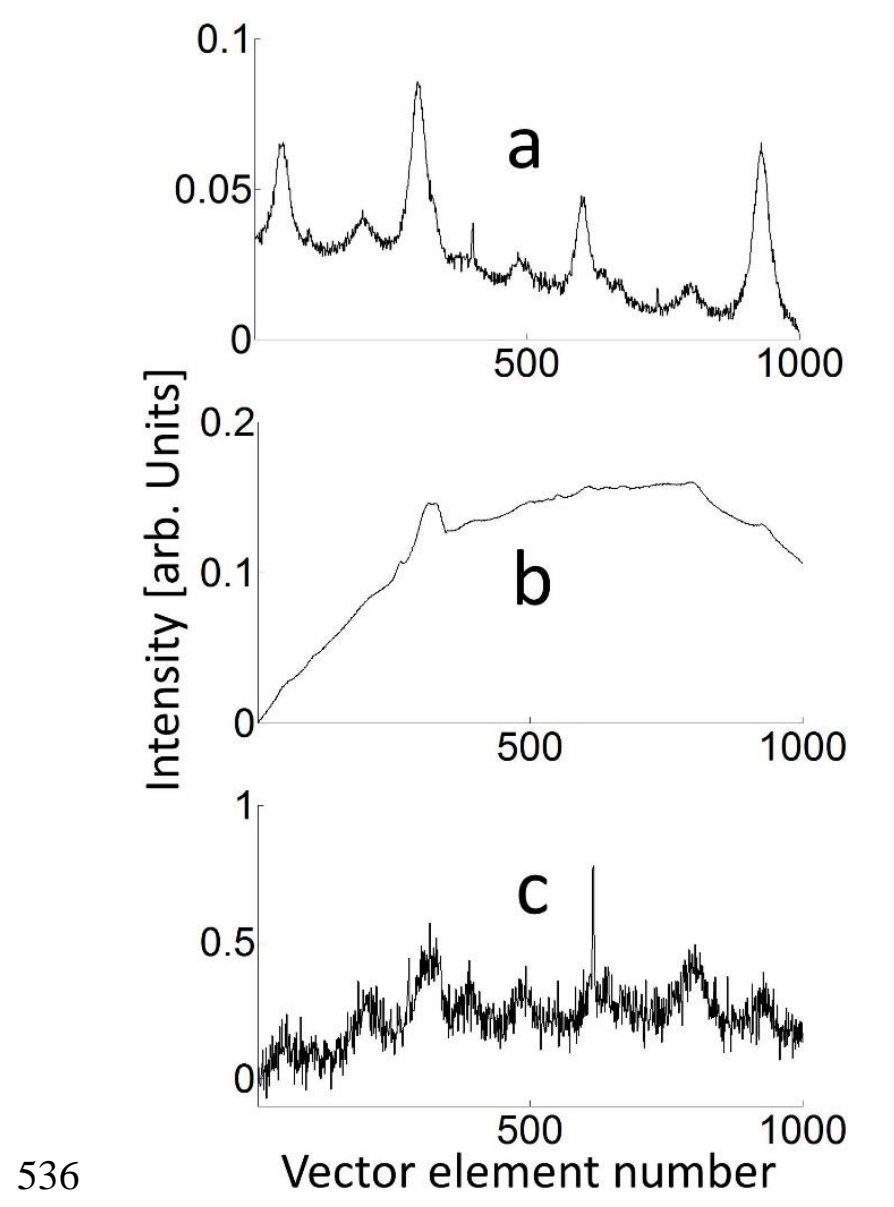

537

538 Figure 2. Examples of simulated spectra after adding baseline distortion, shot noise and 539 multiplying the total intensity: (a) mixture $\mathrm{n}$. 1 , linear baseline (BSR $=0.5), \mathrm{SNR}=30$,

540 norm $=1$; (b) mixture n. 4, polynomial baseline $(\mathrm{BSR}=4), \mathrm{SNR}=100$, norm $=4$; $(\mathrm{c})$

541 mixture n. 9, polynomial baseline $(\mathrm{BSR}=0.5), \mathrm{SNR}=5$, norm $=8$. For abbreviations see 542 Table 1. 

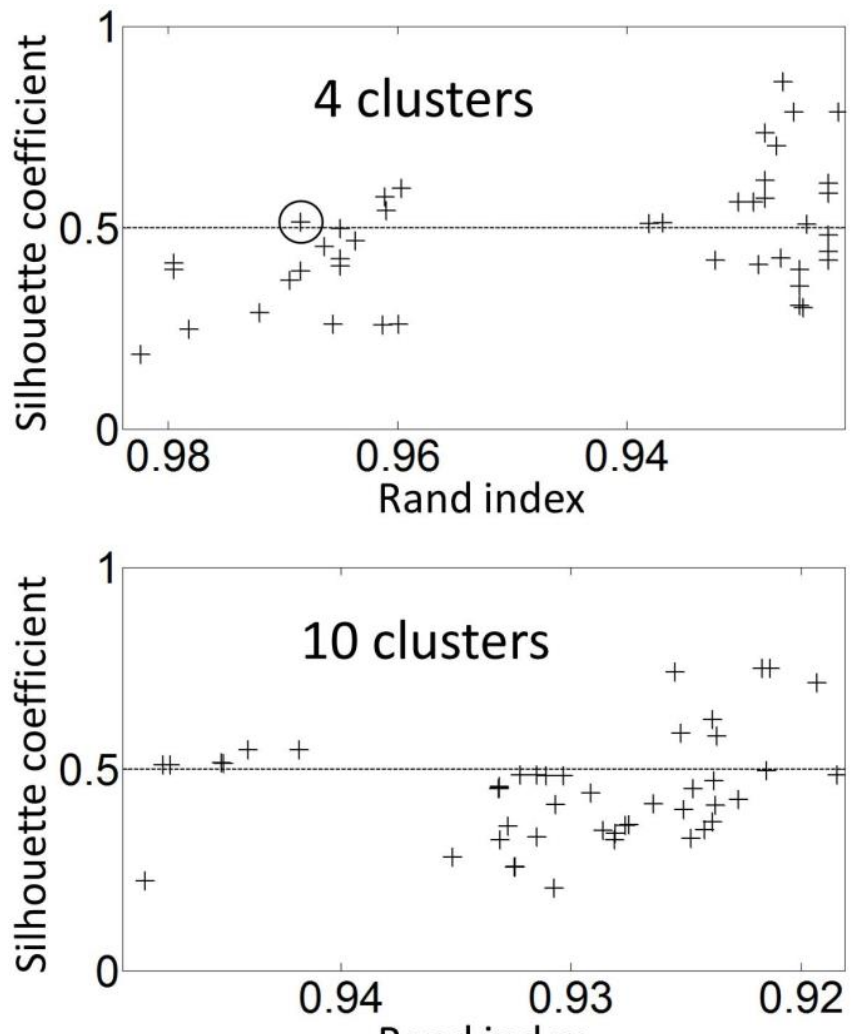

Rand index

545 Figure 3. Plot of $R$ vs. $S$ for the 50 most accurate clusterings by the $k$-means method with

$546 k=4$ (top) and $k=10$ (bottom), respectively. The dashed line marks $S=0.5$; the circle in 547 the top plot highlights the $7^{\text {th }}$ most accurate clustering. 

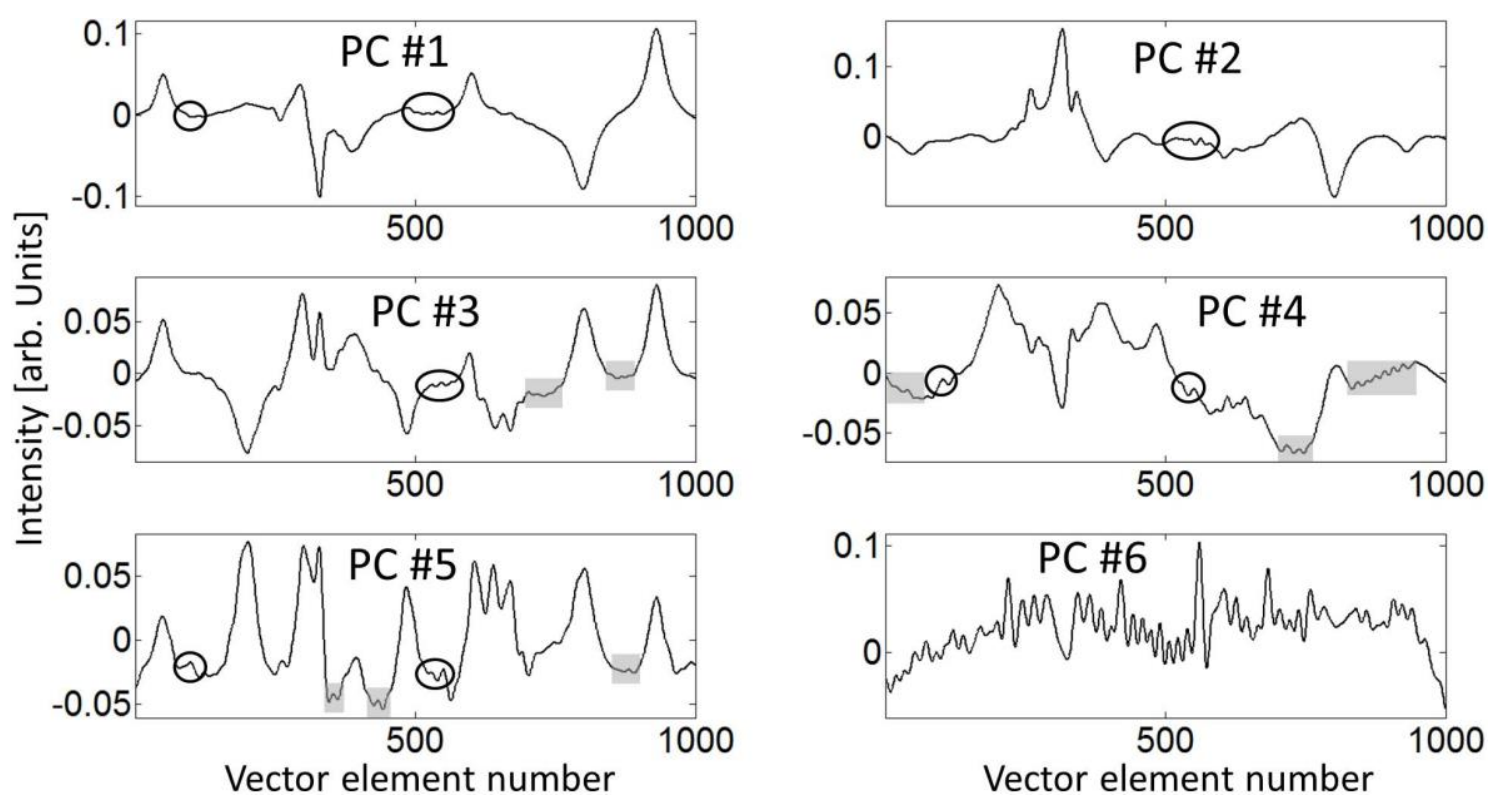

549

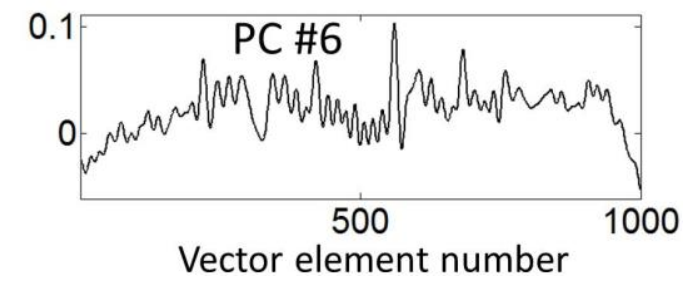

550 Figure 4. Plot of the first 6 PCs after WPD, WavS and 1N. These PCs explain $87 \%$ of

551 data variance; $80 \%$ and $90 \%$ are spanned by 4 and 11 PCs, respectively. Spectral features

552 from the 'trace' component $\mathrm{E}$ (ovals) are mixed with and hardly distinguishable from

553 several noise-related ones (gray shading). 


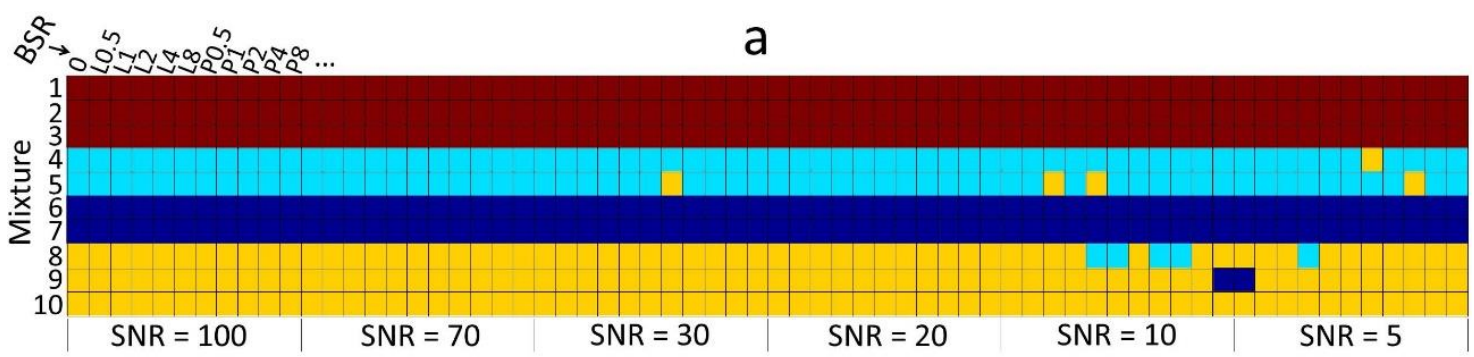

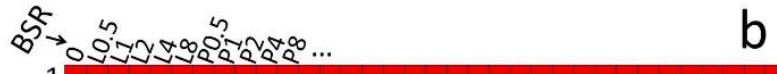

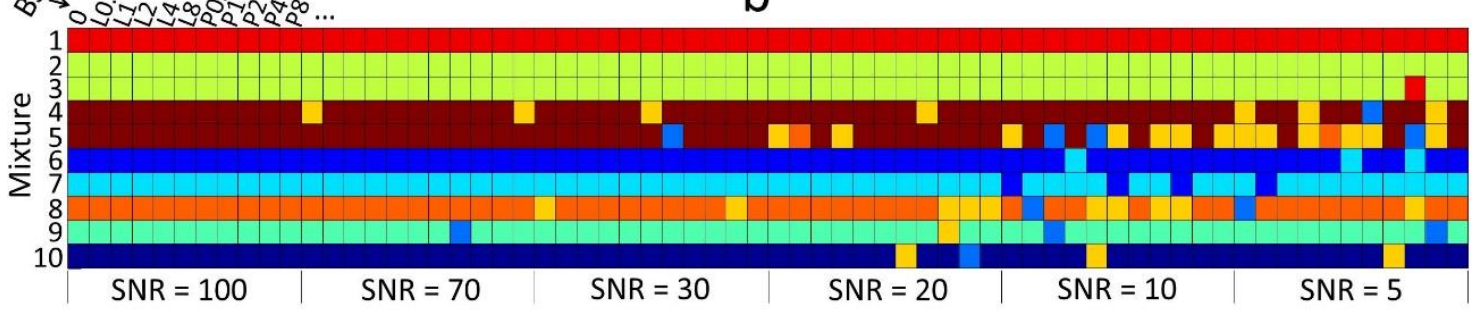

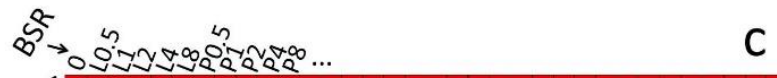

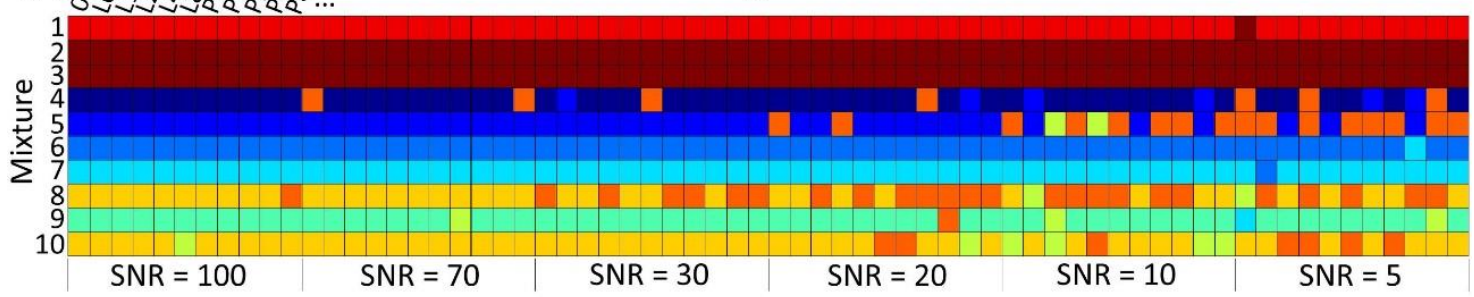

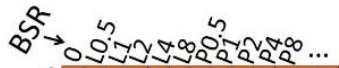

d

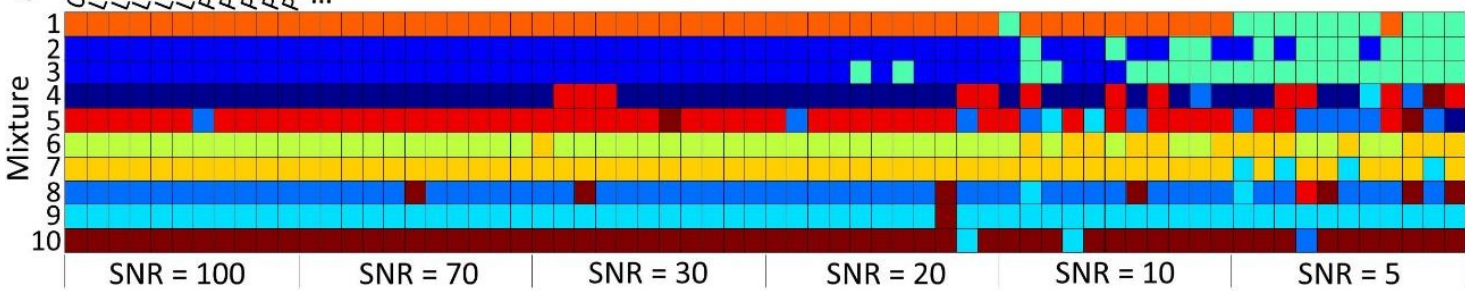

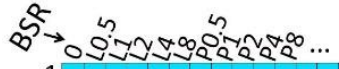

e

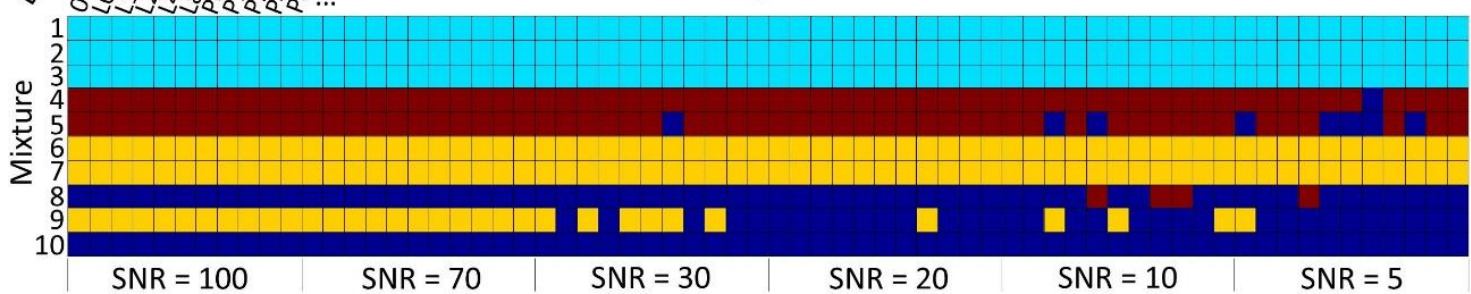


556 Figure 5. Color-based visualization of clusterings obtained from certain pre557 processing/clustering combinations. Each square represents a data point, identified by the 558 mixture number, the SNR (increasing in blocks from left to right) and the BSR preceded 559 by "L" or "P" for linear and polynomial baseline, respectively (the BSR sequence is 560 repeated for each SNR block). The norm is not indicated for simplicity, since all these

561 combinations include a normalization step that cancels the intensity variations. Figure 5a:

$562\{\mathrm{KMC}, k=4, \mathrm{WPD}, \mathrm{NoS}, 2 \mathrm{~N}, 90 \%[76], \mathrm{CB}\}$ (highest $R$ ). Figure 5b: $\{\mathrm{KMC}, k=10$,

563 WPD, NoS, 1N, noPCA, cos $\}$ (highest $R$ with 10 clusters). Figure 5c: $\{$ AHC, $k=10$,

564 WPD, WavS, 2N, 80\%[4] $\}$ (highest $R$ by AHC with 10 clusters). Figure $5 \mathrm{~d}$ : $\{$ KMC, $k=$ $56510, \mathrm{WPD}, \mathrm{WhS}, 1 \mathrm{~N}, 80 \%[3], \mathrm{CB}$ ( $7^{\text {th }}$ highest $R$ by KMC with 10 clusters). Figure 5e: $566\{$ KMC, $k=4, \mathrm{WPD}, \mathrm{WhS}, \mathrm{N} 2,80 \%[4], \mathrm{CB}\}\left(R=0.938,21^{\text {st }}\right.$ highest $R$ by KMC with 4 567 clusters). For abbreviations see Table 2. 


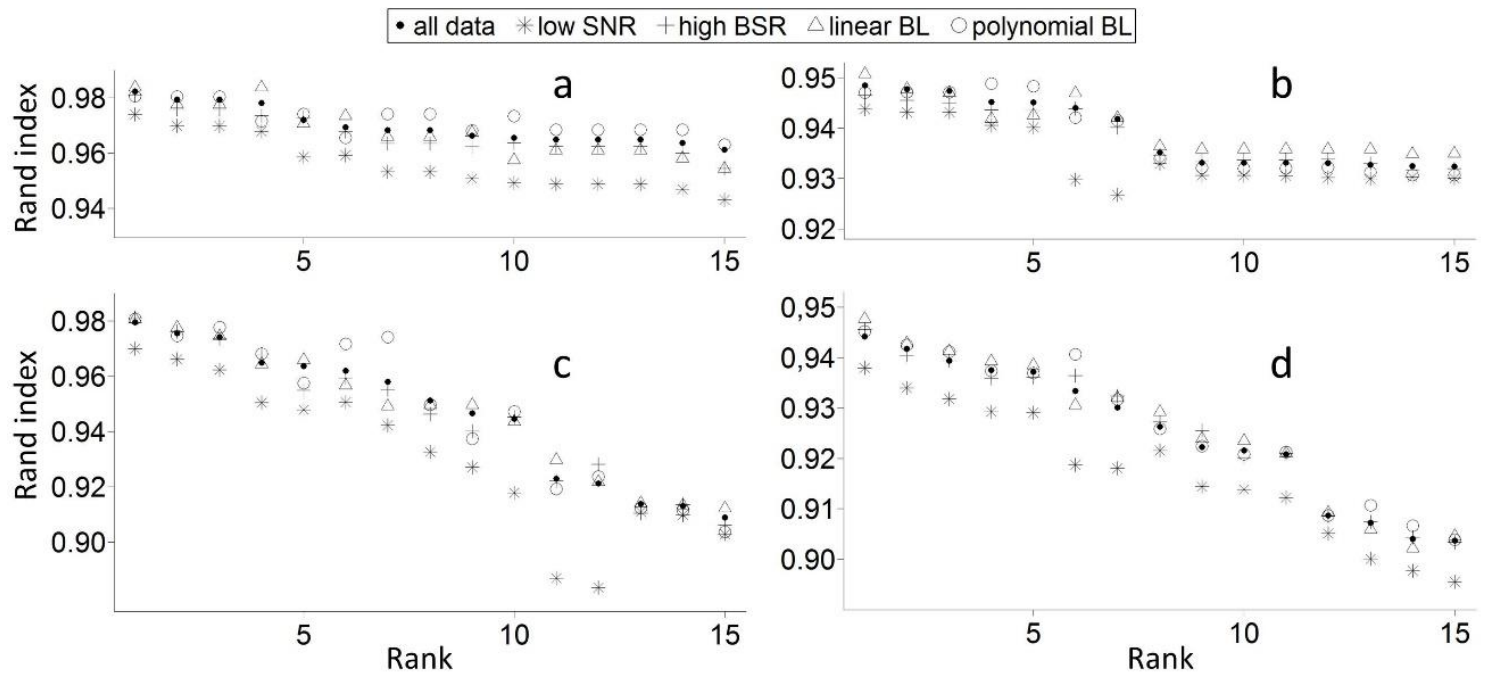

570 Figure 6. Rand statistics of the pre-processing/clustering combinations from Table 3 for

571 all data (filled dots) and for the following subsets: spectra with SNR = 30, 20, 10, 5 (stars);

572 spectra with BSR $=2,4,8$ (crosses); spectra with linear baselines (triangles); spectra with

573 polynomial baselines (circles). Figure 6a: KMC, $k=4$; Figure 6b: KMC, $k=10$; Figure

574 6c: AHC, $k=4$; Figure 6d: AHC, $k=10$; 

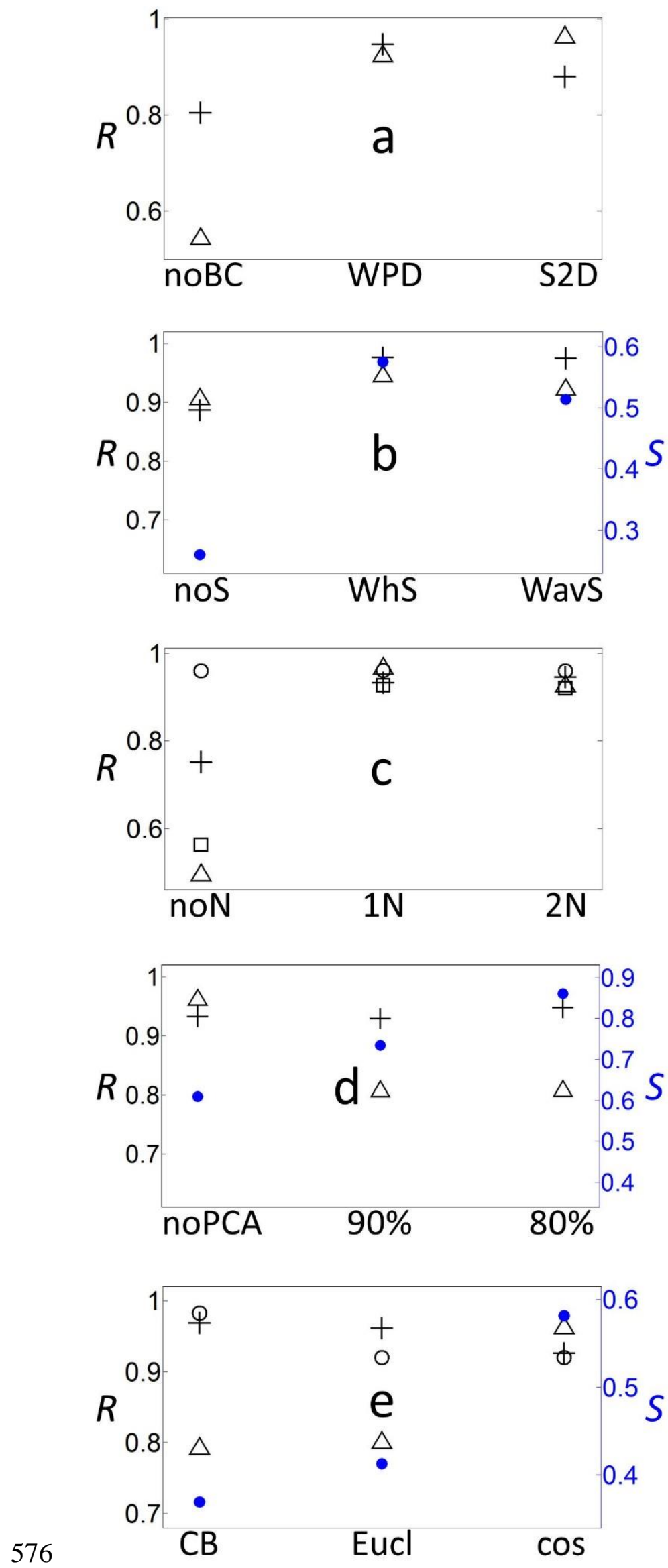
577 Figure 7. $R$ and $S$ trends in selected sections of the solution space focusing on a single 578 pre-processing/clustering variable. The examples are all taken from KMC results, except 579 where specified. Figure 7a, dependence on the baseline correction method: $R$ for $\{k=10$, $580 \mathrm{WhS}, 2 \mathrm{~N}, 80 \%$, Eucl $\}$ (triangles) and $\{k=4, \mathrm{WhS}, 1 \mathrm{~N}$, noPCA, cos $\}$ (crosses). Figure $5817 \mathrm{~b}$, dependence on the smoothing method: $R$ for $\{k=10$, WPD, $1 \mathrm{~N}, 80 \%$, Eucl $\}$ 582 (triangles) and $\{\mathrm{AHC}, k=4$, WPD, $1 \mathrm{~N}, 90 \%\}$ (crosses); $S$ for $\{k=4$, WPD, $1 \mathrm{~N}, 80 \%$, 583 CB $\}$ (filled blue circles). Figure 7c, dependence on the normalization method: $R$ for $\{k=$ 10, WPD, WavS, 80\%, CB $\}$ (crosses), $\{k=4$, WhS, S2D, noPCA, cos $\}$ (circles), $\{k=4$, 585 WPD, WavS, 90\%, $\cos \}$ (squares) and $\{k=4$, WPD, WhS, 90\%, Eucl $\}$ (triangles). Figure $5867 \mathrm{~d}$, dependence on the PC compression: $R$ for $\{k=10$, WPD, WhS, 2N, Eucl $\}$ (crosses) 587 and $\{k=4, \mathrm{WhS}, \mathrm{S} 2 \mathrm{D}, 1 \mathrm{~N}, \cos \}$ (triangles); $S$ for $\{k=4, \mathrm{WPD}, \mathrm{WhS}, 1 \mathrm{~N}, \cos \}$ (filled 588 blue circles). Figure 7e, dependence on the distance metric: $R$ for $\{k=4$, WPD, WavS, $1 \mathrm{~N}, 80 \%[4]\}$ (crosses), $\{k=4, \mathrm{WhS}, \mathrm{S} 2 \mathrm{D}, 1 \mathrm{~N}$, noPCA $\}$ (triangles) and $\{k=4$, WDP, noS, 2N, 90\%[76] $\}$ (circles); $S$ for $\{k=10$, WPD, WavS, $1 \mathrm{~N}, 90 \%\}$ (filled blue circles).

591 For abbreviations see Table 2. 